\title{
Cilia regulate meiotic recombination in zebrafish
}

Haibo Xie ${ }^{1,3,4}$, Xiaosi Wang ${ }^{2}$, Minjun Jin ${ }^{1,4}$, Lanqin $\mathrm{Li}^{1,4}$, Junwen $\mathrm{Zhu}^{2,5}$, Yunsi

Kang $^{1,4}$, Zhe Chen ${ }^{1}$, Yonghua Sun ${ }^{2,5 *}$ and Chengtian Zhao ${ }^{1,3,4 *}$

${ }^{1}$ Institute of Evolution \& Marine Biodiversity, Ocean University of China, Qingdao, 266003, China

${ }^{2}$ State Key Laboratory of Freshwater Ecology and Biotechnology, Institute of Hydrobiology, Innovation Academy for Seed Design, Hubei Hongshan Laboratory, Chinese Academy of Sciences, Wuhan 430072, China

${ }^{3}$ Laboratory for Marine Biology and Biotechnology, Qingdao National Laboratory for Marine Science and Technology, Qingdao, 266003, China

${ }^{4}$ Sars-Fang Centre, Ministry of Education Key Laboratory of Marine Genetics and Breeding, College of Marine Life Sciences, Ocean University of China, Qingdao 266003, China

${ }^{5}$ College of Advanced Agricultural Sciences, University of Chinese Academy of Sciences, Beijing 100049, China.

*Corresponding authors.

Email:

chengtian zhao@ouc.edu.cn (C.Z.)

yhsun@ihb.ac.cn (Y.S.) 


\begin{abstract}
Meiosis is essential for evolution and genetic diversity in almost all sexual eukaryotic organisms. The mechanisms of meiotic recombination, such as synapsis, have been extensively investigated. However, it is still unclear whether signals from the cytoplasm or even outside of the cell can regulate the meiosis process. Cilia are microtubule-based structures that protrude from cell surface and function as signaling hubs to sense extracellular signals. Here, we reported an unexpected and critical role of cilia during meiotic recombination. During gametogenesis of zebrafish, cilia were specifically present in the prophase stages of both spermatocytes and primary oocytes. By developing a novel germ cellspecific CRISPR/Cas9 system, we demonstrated that germ cell-specific depletion of ciliary genes resulted in compromised double strand break repair, reduced crossover formation, and increased germ cell apoptosis. Our study reveals a previously undiscovered role for cilia during meiosis and suggests that extracellular signals may regulate meiotic recombination via this particular organelle.
\end{abstract}




\section{Introduction}

Meiosis is probably the most key event in the life cycle of sexually reproducing eukaryotes. During the prophase of the first meiotic division, homologous chromosomes (homologs) of the maternal and paternal origins pair and synapse, which creates a context for the exchange of genetic information between homologs through an elaborate process, homologous recombination (HR). Meiotic recombination has been extensively studied in different model systems ranging from yeast, protists to mammals (Loidl, 2016; Zelkowski et al, 2019). In a classic HR model, DNA doublestrand breaks (DSBs) are induced by the meiotic topoisomerase-like protein SPO11, then the phosphorylation of histone variant H2A.X is induced around the DSB sites. Meanwhile, DSB ends are processed and the resulting single strand overhangs are bound by recombinases including Dmc1 and Rad51. The broken DNA ends and these proteins form nucleofilaments and further invade the homologous template for homology searching and HR. Dmc1-mediated homology searching also promotes the formation of the synaptonemal complex (SC), an evolutionarily conserved tripartite protein structure formed between paired homologs. The SC is indispensable for multiple meiosis processes, including enhancing the interhomolog recombination, ensuring obligate crossover (CO) formation, and thus, safeguarding the faithful segmentation of chromosomes (see (Zickler \& Kleckner, 2015)). Depending on the resolution of the double holiday junction, repairing of DSBs will be channeled to MutL homolog 1 (Mlh1)-dependent pro-CO pathway or non-CO pathways. The crossover recombination event leads to the reciprocal exchange of alleles between two homologues, thus ensures the genetic diversity of the progeny (Handel \& Schimenti, 2010).

One of the key aspects of the meiotic recombination is the reorganization of the chromosome during prophase to ensure homologous pairing. The formation of the telomere bouquet is an evolutionarily conserved mechanisms for promoting homologous pairing (Loidl, 2016). Telomeres are attached to the nuclear envelope (NE) and clustered to one side of the nuclear through the linker of nucleoskeleton and 
cytoskeleton (LINC) complex, which bridges the movement of chromosome to the dynein motor moving on the perinuclear microtubules (Burke, 2018). Intriguingly, telomeres are usually clustered at the NE near the microtubule organizing center (MTOC), suggesting a potential role of the microtubule network in promoting the homologous pairing (Elkouby et al, 2016; Sato et al, 2009; Sawin, 2005). Although the mechanisms of homologous recombination has been extensively studied, it is still an enigma whether the meiotic recombination can be modulated by signals from the cytoplasm or extracellular environment.

\section{Results and discussion}

Cilia are microtubule-based structures that are nucleated from the basal body, a highly conserved structure acting as the MTOC. The sperm flagellum is a special type of long motile cilia that is essential for sperm motility (Fig 1A). Unexpectedly, when examining the development of zebrafish sperm flagella, we observed a special cilia-like structure that were present in the spermatocytes (Fig 1A). Double immunostaining with antiacetylated tubulin (a cilia marker) and SYCP3 (a lateral element of SC) antibodies showed that these ciliary structures were initially formed at leptotene stage, and gradually elongated until diplotene stage (Fig 1A). The diplotene cilia was about half length of sperm flagella (Fig 1A-B). Interestingly, these cilia were disappeared at diakinesis stage, implying that they are not the rudiments of sperm flagella. Further immunostaining with the centrosomal marker, anti- $\gamma$ tubulin antibody, indicated that these microtubules were nucleated from one of the centriole (Fig 1C, S1). Moreover, these cilia can also be labeled with anti-glutamylated tubulin antibody (Fig S2A). Whereas, although sperm flagella were positive for both mono- or poly-glycylated tubulin antibody, these spermatocyte cilia were lack of this kind of tubulin modifications (Fig S2B). Furthermore, ultrastructural analysis showed that these cilia had a " $9+0$ " axonemal configuration, in contrast to the " $9+2$ " arrangement of sperm flagella (Fig 1D-F'). Finally, this special type of cilia was also present in the leptotene and zygotene stages of primary oocytes (Fig S3). 
The presence of these cilia during prophase I of the first meiotic division implies that cilia may participate in the regulation of homologous recombination. As cilia are essential for early zebrafish embryos development (Song et al, 2016), we seek to design a strategy to knockout these cilia specially in the spermatocytes. To reach this goal, we developed a germ cell-specific CRISPR/Cas9 system by generating a primordial germ cell (PGC)-specific Cas9-transgenic line, $\operatorname{Tg}$ (kop:cas9-p2a-egfp-UTRnanos3) (for abbreviation: $\operatorname{Tg}$ (kop:cas9-UTRnanos3)) (Fig 2A), in which a Cas9-2A-EGFP expression cassette was placed under the control of the PGC-specific askopos (kop) promoter and nanos3 3'-UTR (Xiong et al, 2013). Since the Cas9 protein was expressed only in the PGCs of $\operatorname{Tg}$ (kop:cas9-UTRnanos3) embryos, injection of gRNA into the embryos would not result in somatic mutation but in PGCs-specific gene knockout. Further crossbreeding of the injected F0 fish could generate maternal zygotic mutants in the F1 generation, even if the zygotic mutants were lethal (Fig S4A). To test the efficacy and penetrance of PGCs-specifically expressed Cas9 in the $\operatorname{Tg}(\mathrm{kop}$ :cas 9 UTRnanos3) embryos, we first injected a validated sgRNA targeting tcf7lla (Zhang et al, 2020), whose maternal zygotic mutants (MZtcf7lla), but not maternal (Mtcf7lla) or zygotic mutants (Ztcf7lla), shows a headless phenotype (Kim et al, 2000). Crossing two high-efficient germline mutated females and males resulted in a typical headless phenotype in more than $50 \%$ of the F1 embryos (Fig S4B, C), suggesting the highefficiency of PGCs-expressed Cas9. Similarly, we were also able to generate MZpou5f3 mutants which exhibited severe developmental defects (Reim \& Brand, 2006; Schulz \& Harrison, 2019) (Fig S4 D,E). These data demonstrate that the Tg(kop:cas9UTRnanos3) line expresses a highly efficient Cas9 protein which enables germ cellspecific gene knockout. Noticeably, albeit with high efficiency, the penetrance of mutations in the gametes was not $100 \%$, which may be due to the temporal presence of sgRNAs.

To further improve the efficiency of gene editing in the PGCs, we generated a stable $\operatorname{Tg}(U 6: 3 x s g R N A-k i f 3 a)$ transgenic line, in which three sgRNAs targeting zebrafish kif3a was each driven by an individual U6 promoters in tandem. Kif3a is a plus-end 
motor protein crucial for cilia assembly (Zhao et al, 2012). We further crossed the $\operatorname{Tg}$ (kop:cas9-UTRnanos3) line with the $\operatorname{Tg}(U 6: 3 x s g R N A-k i f 3 a)$ line (Fig 2B) to generate the $\operatorname{Tg}(k o p$ :cas9-UTRnanos3; U6:3xsgRNA-kif3a) double transgenic line. By crossing the double transgenic female with kif3a heterozygotic male, we have successfully generated maternal-zygotic kif3a mutants (MZkif3a). Around $50 \%$ of embryos (340 of 707) displayed body curvature defects, strongly implying that Kif3a was deficient in almost all of the eggs produced from the double transgenics. Compared with Zkif3a mutants, MZkif3a mutants displayed strong body curvature defects, together with the increased size of the brain ventricles, a typical feature of hydrocephalous (Fig 2C-F) (Feng et al, 2017). Further whole mount staining results confirmed that cilia were completely absent in the MZkif3a mutants, while cilia were still formed in the spinal cord and otic vesicle of Zkif3a mutants at early stages due to the maternal effects of Kif3a (Fig 2G). As for the double transgenic males, although they developed to adulthood normally as the wildtype males, they could not fertilize wildtype eggs due to the germ-cell disruption of Kif3a (Fig 3A-C). The spermatozoa were able to be produced in the testis, while all the flagella were significantly shorter than those of control siblings (Fig 3D-E, S5A). These data also suggest that sperm flagellum can still be partially assembled in the absence of Kif3a.

Since Cas9 protein was expressed in PGCs, it is rationale that kif3a gene should be disrupted at earlier stages. Indeed, cilia failed to form in the primary spermatocytes, and the staining of acetylated tubulin was only detected in the centrosomes (Fig 3F). Further staining with SYCP3 on the sections of testis showed that cilia were completely absent in all the earlier stage primary spermatocytes (Fig S5B), which further confirmed the nearly $100 \%$ efficiency in inducing germ cell-specific knockout of kif3a (gcKOkif3a) by the double transgenic approach (Fig 2B). Noticeably, strong signals were still present in those regions that were rich for spermatozoa, which were related to the remaining shorter sperm flagella (Fig S5B). Moreover, cilia in the primary oocytes were also absent in the double transgenic females (Fig S3).

During meiotic recombination, the formation of DSB provokes a rapid DNA damage 
response, leading to the phosphorylation of a histone $\mathrm{H} 2 \mathrm{~A}$ isoform at sites of DSBs. By using the anti- $\gamma$-H2A.X antibody, we can distinguish spermatocytes at both leptotene and zygotene stages. The staining of $\gamma-\mathrm{H} 2 \mathrm{~A} . \mathrm{X}$ was gradually disappeared from midzygotene stage (Xie et al, 2020). The staining signals were similar between gcKOkif3a spermatocytes and control siblings at leptotene stage, while unexpectedly, the spermatocytes had strong $\gamma$-H2A.X staining throughout their nuclei at zygotene stage in gcKOkif3a mutants (Fig 4A-B). By counting the number of $\gamma$-H2A.X positive germ cells, we further found that the number of spermatocytes within each spermatocyst was comparable between the gcKOkif3a and control fish (Fig 4C), suggesting that loss of spermatocyte cilia did not affect the mitotic division of spermatogonium.

The strong signals of $\gamma-\mathrm{H} 2 \mathrm{~A} . \mathrm{X}$ staining suggest that DSB repair may be affected in the absence of spermatocyte cilia. Next, we checked the distribution of Rad51, one of the key recombinases recruited to the break sites for DSB repair. Compared with those of control cells, Rad51 signals decreased substantially in leptotene-stage spermatocytes of gcKOkif3a males (Fig 4D-E). Rad51 signals became weaker at zygotene stage and there were no difference in the staining of Rad51 between mutant and control siblings at later zygotene stage (Fig 4D). Rad51 is required for the formation of crossovers. Next, we evaluated the crossover events by investigating the number of Mlh1 foci on surface spread spermatocytes. The number of Mlh1 foci decreased significantly in the mutant spermatocytes (Fig 4F-G). Finally, TUNEL assay results indicated that mutant testis contained high number of apoptotic spermatocytes than those of control siblings (Fig 4H-I). Altogether, these data suggested that loss of spermatocyte cilia affected the DSB repair process, leading to decreased crossover formation and cell apoptosis.

Finally, to exclude the non-ciliary role of Kif3a during meiotic progression, we further generated $\operatorname{Tg}(h s p 70 l: i f t 88$-egfp) transgenic line in which ift88, another essential gene for ciliogenesis (Tsujikawa \& Malicki, 2004), was driven by a heat shock promoter. By performing routine heat shock, we were able to rescue ovl(ift88) mutants with this transgene (Fig S6A). Interestingly, the adult mutants can still survive for a short period even without heat inducement. By comparing mutants between heat induced and 
untreated fish, we found that the spermatocyte cilia were lost in the testis of non-heatshocked controls (Fig S6B-C). Moreover, mutants generated from these non-heatshocked adult females and heterozygotic males showed stronger body curvature and ciliogenesis defects, resembling those of MZovl embryos (Fig S7) (Huang \& Schier, 2009). Similar to those of kif3a mutants, loss of ift 88 also caused abnormal cell apoptosis of the spermatocytes (Fig S6D-E), further confirming the role of cilia during meiosis processing.

In summary, here we reported a novel type of cilia existing in the primary spermatocytes and oocytes during meiotic recombination. By developing a germ cellspecific gene knock out approach, our study demonstrates that depletion of these early germ cell-specific cilia results in compromised processing of DSB repair, impaired crossover formation, and increased cell apoptosis as well (Fig 4J). It is noteworthy that oocytes and spermatozoa could be still produced in the absence of germ cell-specific cilia, albeit with cell apoptosis and flagellar defects in the spermatozoa. It is likely that this cilium may regulate DSB repair to ensure crossover formation, whereas it is not essential for the processing of meiosis. Cilia act as a cellular antenna to sense extracellular signals (Ishikawa \& Marshall, 2011). Although we do not know yet the detailed mechanisms of these cilia in the regulation of meiotic recombination, our data provide an important message that meiotic recombination can be regulated by signals outside the nucleus. These cilia may function as signal hubs to sense signals outside the cell that help recruit DSB repair components, such as Rad51 and DMC1, to the DSB sites and ensure the HR processing. On the other hand, these cilia are nucleated from the basal body, from which the bouquet microtubule originates. It is possible that the cilia may supply forces for dynein/kinesin motors to support telomere movement and thus homolog pairing (Fig 4J). Further experiments are needed to dissect the precise signaling molecules underlying this intriguing organelle, and the germ cell-specific CRISPR/Cas9 system developed in present study may provide a powerful tool to investigate this process. 


\section{Materials and methods}

\section{Ethics statement}

All zebrafish study was conducted according standard animal guidelines and approved by the Animal Care Committee of Ocean University of China and the Animal Care Committee of the Institute of Hydrobiology, Chinese Academy of Sciences.

\section{Zebrafish strains}

All zebrafish strains were maintained at 14-hour light /10-hour dark cycle at $28.5^{\circ} \mathrm{C}$. The plasmid for generating $\operatorname{Tg}(k o p$ :cas9-p2a-egfp-UTRnanos3) transgenic fish was generated by replacing the egfp sequence in the pTol2(kop:egfp-UTRnanos3) construct (Xiong et al., 2013), with an in frame sequence, cas9-p2A-egfp, which encodes zebrafish codon optimized Cas9, p2A peptide, and EGFP. The plasmids for $\operatorname{Tg}(U 6 x: 3 x \operatorname{sgRNA-kif3a)}$ transgene was generated by Golden Gate cloning as previously described (Yin et al, 2016). Three single guide RNAs (sgRNAs) targeting kif3a (sgRNA1, GGGAAAACGTTCACTATGGA; sgRNA2, GGCCAAACTCGACATGGAGG; sgRNA3， GAGGAGGTGAGAGATCTGTT) were each driven by distinct zebrafish U6 promoters. The $\operatorname{Tg}(U 6 x: 3 x \operatorname{sg} R N A$-kif3a $)$ transgenic fish were further crossed to $\operatorname{Tg}(k o p$ :cas $9-p 2 a-e g f p$-UTRnanos 3) transgenic line to get the double transgenic fish. The pTol2-hsp70l:ift88-egfp plasmid was constructed through Multisite Gateway cloning and injected into the homozygous ovl (ift88) mutants at one cell stage. The injected embryos were heat induced for three hours every day until adulthood for further analysis.

\section{Microinjection of single guide RNA (sgRNA)}

For microinjection based primordial germ cell targeted gene knock experiment, single guide RNAs against $t c f 7 l 1 a$ and pou5f3 were GGAGGAGGAGGTGATGACCT and GGGTGAACTACTACACGCCA respectively, as previously described (Zhang et al., 2020). The sgRNAs were injected with a dosage of 80 pg per embryo into the 
$\operatorname{Tg}(k o p: c a s 9-p 2 a-e g f p-U T R n a n o s 3)$ transgenic embryo at 1-cell stage.

\section{Chromosome spreading and immunostaining}

Chromosome spreading steps were similar to previously published (Xie et al., 2020). Briefly, the dissected testis was first incubated in $50 \mu \mathrm{l} 1 \mathrm{X}$ PBS for $5 \mathrm{~min}$, then transferred into $300 \mu \mathrm{l}$ hypotonic solution $(100 \mu \mathrm{l}$ 1xPBS plus $200 \mu \mathrm{l}$ double distilled water) for $25 \mathrm{~min}$ on an adhesive slide. After fixation with $4 \%$ paraformaldehyde (PFA) for $5 \mathrm{~min}$, the cells were washed three times with PBS, blocked with blocking solution (10\% goat serum, $3 \% \mathrm{BSA}, \quad 0.05 \%$ TritonX-100) for $30 \mathrm{~min}$, then followed by regular antibody staining.

\section{Immunofluorescence and TUNEL assay}

Zebrafish testes were first dissected and fixed in 4\% PFA overnight at $4{ }^{\circ} \mathrm{C}$. After three times wash with PBS for 10 min each, the testes were infiltrated in $30 \%$ sucrose overnight at $4{ }^{\circ} \mathrm{C}$, then embedded with tissue freezing medium (OCT). Cryosectioning was performed using LEICA cryostat (CM1860). TUNEL assay was performed using the in situ cell death detection kit (Roche) according to standard protocols from the manufacturer. Images were collected with a Leica Sp8 confocal microscope.

To visualize cilia in the primary ovaries, zebrafish females about 1.5 month old were first euthanized with ice-cold water, and ovaries were dissected and transferred into 4\% PFA overnight at $4{ }^{\circ} \mathrm{C}$. After three times wash with PBST ( 1 X PBS $+0.1 \%$ tween 20$)$, the ovaries were further blocked with Blocking Solution (0.3\% TritonX-100, 10\% Fetal Bovine Serum, 1xPBS) for $1.5 \mathrm{~h}$ at room temperature. Antibody staining steps were similar to those of testes.

For immunofluorescence, the following antibodies were used: mouse anti-acetylated tubulin, rabbit anti-SYCP3, rabbit anti- $\gamma$-H2A.X, rabbit anti-RAD51, mouse antiMLH1, mouse anti- $\gamma$-tubulin, rabbit anti-acetylated tubulin, mouse anti-glutaminated tubulin, mouse anti-monoglycylated tubulin and mouse anti-polyglycylated tubulin. 


\section{Immunohistochemical staining}

Zebrafish testes were dissected and fixed in $4 \%$ PFA overnight at $4{ }^{\circ} \mathrm{C}$. After gradual dehydration through $30 \%, 50 \%, 75 \%, 95 \%$ and $100 \%$ ethanol, the testes were embedded in JB4 embedding medium (Polysciences Inc., Warrington, PA, USA). Transverse sections through the testes were collected using Leica RM2235 microtome and stained with hematoxylin and eosin. Images were taken using a Leica DM2500 microscope.

\section{Ultrastructural analysis}

Zebrafish testis were dissected and fixed overnight in $2.5 \%$ glutaraldehyde. The testis was washed three times in PBS for 10 min each, fixed again in $1 \%$ osmium tetroxide. After that, the testis was washed, gradually dehydrated to $100 \%$ acetone, and then embedded in Epon812 resin. Ultrathin sections were collected and stained with uranyl acetate and lead citrate. The sections were observed on a transmission electron microscope (JEM-1200EX) and imaged with Olympus Soft Imaging Solutions.

\section{Statistical analysis}

All the confocal images were captured using Leica Sp8 confocal microscope. To compare the fluorescence signals between control and mutants, all the images were collected at the same parameters within each experiments. All experiments were repeated at least three times. Statistical analysis was performed using ImageJ, Microsoft Excel or GraphPad Prism 6 software. All data were presented as mean \pm S.D. as indicated in the figure legends. A value of $\mathrm{p}<0.05$ was considered statistically significant.

\section{Acknowledgement}

We thank Dr. Feng Xiong at the China Zebrafish Resource Center for generating the pTol2(kop: cas9-p2a-egfp-UTRnanos3) construct. We thank Dr. Liangran Zhang, Wei 
Li and Ming Shao for providing DNA constructs and reagents. We also thank Dr. Miao Tian and Tao Qiu for their help during the preparation of this manuscript. This work was supported by the National Natural Science Foundation of China (Nos. 31991194, 32025037, 32125015), grant from the Fundamental Research Funds for Central Universities (NO. 201941004) and grant from the State Key Laboratory of Freshwater Ecology and Biotechnology (2019FBZ05).

\section{References}

Burke B (2018) LINC complexes as regulators of meiosis. Curr Opin Cell Biol 52: 22-29

Elkouby YM, Jamieson-Lucy A, Mullins MC (2016) Oocyte Polarization Is Coupled to the Chromosomal Bouquet, a Conserved Polarized Nuclear Configuration in Meiosis. PLoS Biol 14: e1002335

Feng D, Chen Z, Yang K, Miao S, Xu B, Kang Y, Xie H, Zhao C (2017) The cytoplasmic tail of rhodopsin triggers rapid rod degeneration in kinesin-2 mutants. J Biol Chem 292: $17375-17386$

Handel MA, Schimenti JC (2010) Genetics of mammalian meiosis: regulation, dynamics and impact on fertility. Nat Rev Genet 11: 124-136

Huang P, Schier AF (2009) Dampened Hedgehog signaling but normal Wnt signaling in zebrafish without cilia. Development 136: 3089-3098

Ishikawa H, Marshall WF (2011) Ciliogenesis: building the cell's antenna. Nat Rev Mol Cell Biol 12: 222-234

Kim C-H, Oda T, Itoh M, Jiang D, Artinger KB, Chandrasekharappa SC, Driever W, Chitnis AB (2000) Repressor activity of Headless/Tcf3 is essential for vertebrate head formation. Nature 407: 913-916

Loidl J (2016) Conservation and Variability of Meiosis Across the Eukaryotes. Annu Rev Genet 50: 293-316

Reim G, Brand M (2006) Maternal control of vertebrate dorsoventral axis formation and epiboly by the POU domain protein Spg/Pou2/Oct4. Development 133: 2757-2770

Sato A, Isaac B, Phillips CM, Rillo R, Carlton PM, Wynne DJ, Kasad RA, Dernburg AF (2009) Cytoskeletal forces span the nuclear envelope to coordinate meiotic chromosome pairing and synapsis. Cell 139: 907-919

Sawin KE (2005) Meiosis: organizing microtubule organizers. Curr Biol 15: R633635

Schulz KN, Harrison MM (2019) Mechanisms regulating zygotic genome activation. Nature Reviews Genetics 20: 221-234

Song Z, Zhang X, Jia S, Yelick PC, Zhao C (2016) Zebrafish as a Model for Human Ciliopathies. J Genet Genomics 43: 107-120

Tsujikawa M, Malicki J (2004) Intraflagellar transport genes are essential for 
differentiation and survival of vertebrate sensory neurons. Neuron 42: 703-716 Xie H, Kang Y, Wang S, Zheng P, Chen Z, Roy S, Zhao C (2020) E2f5 is a versatile transcriptional activator required for spermatogenesis and multiciliated cell differentiation in zebrafish. PLoS genetics 16: e1008655

Xiong F, Wei ZQ, Zhu ZY, Sun YH (2013) Targeted Expression in Zebrafish Primordial Germ Cells by Cre/loxP and Gal4/UAS Systems. Mar Biotechnol (NY) 15: 526-539

Yin L, Maddison LA, Chen W (2016) Multiplex conditional mutagenesis in zebrafish using the CRISPR/Cas system. Methods Cell Biol 135: 3-17

Zelkowski M, Olson MA, Wang M, Pawlowski W (2019) Diversity and Determinants of Meiotic Recombination Landscapes. Trends Genet 35: 359-370

Zhang F, Li X, He M, Ye D, Xiong F, Amin G, Zhu Z, Sun Y (2020) Efficient generation of zebrafish maternal-zygotic mutants through transplantation of ectopically induced and Cas9/gRNA targeted primordial germ cells. Journal of genetics and genomics = Yi chuan xue bao 47: 37-47

Zhao C, Omori Y, Brodowska K, Kovach P, Malicki J (2012) Kinesin-2 family in vertebrate ciliogenesis. Proc Natl Acad Sci U S A 109: 2388-2393

Zickler D, Kleckner N (2015) Recombination, Pairing, and Synapsis of Homologs during Meiosis. Cold Spring Harbor perspectives in biology 7: a016626 


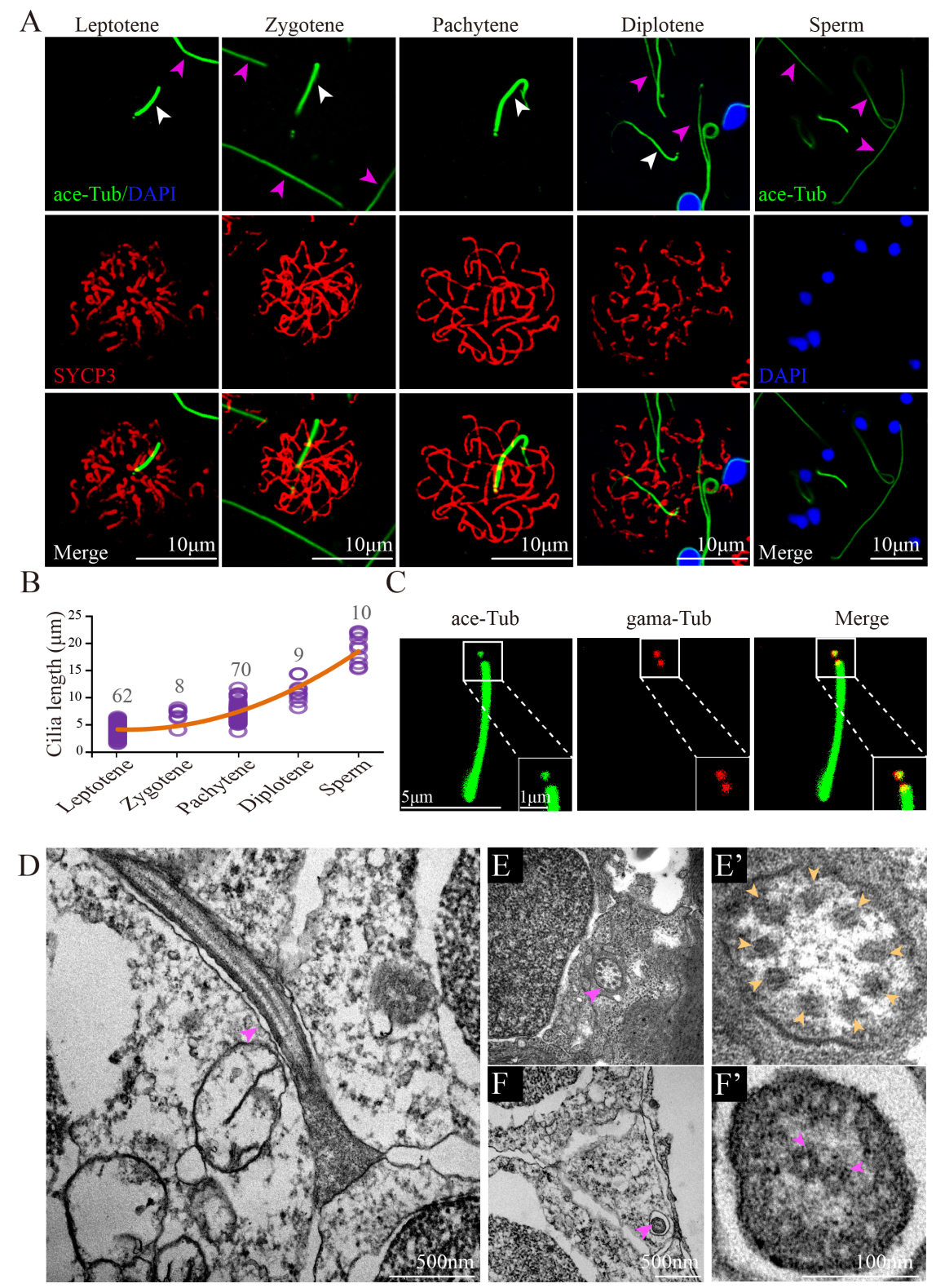

Fig 1. Cilia of primary spermatocytes in meiosis

(A) Confocal images showing cilia (white arrowhead) and sperm flagella (pink arrowhead) labeled with anti-acetylated tubulin (ace-Tub, green) antibody. The synaptonemal complexes were labeled with SYCP3 (red) and nuclei were counterstained with DAPI in blue. (B) Statistical results showing the length of cilia in different stages of primary spermatocytes and sperms. (C) Confocal images showing cilia and basal bodies labeled with anti-acetylated tubulin (ace-Tub, green) and anti- $\gamma$ tubulin (gama-Tub, red) antibodies on primary spermatocyte. (D-F') TEM results showing the ultrastructure of primary spermatocyte cilia (D-E') and sperm flagella (F-F'). Cross section showing the " $9+0$ " configuration of spermatocyte cilia (E'). 

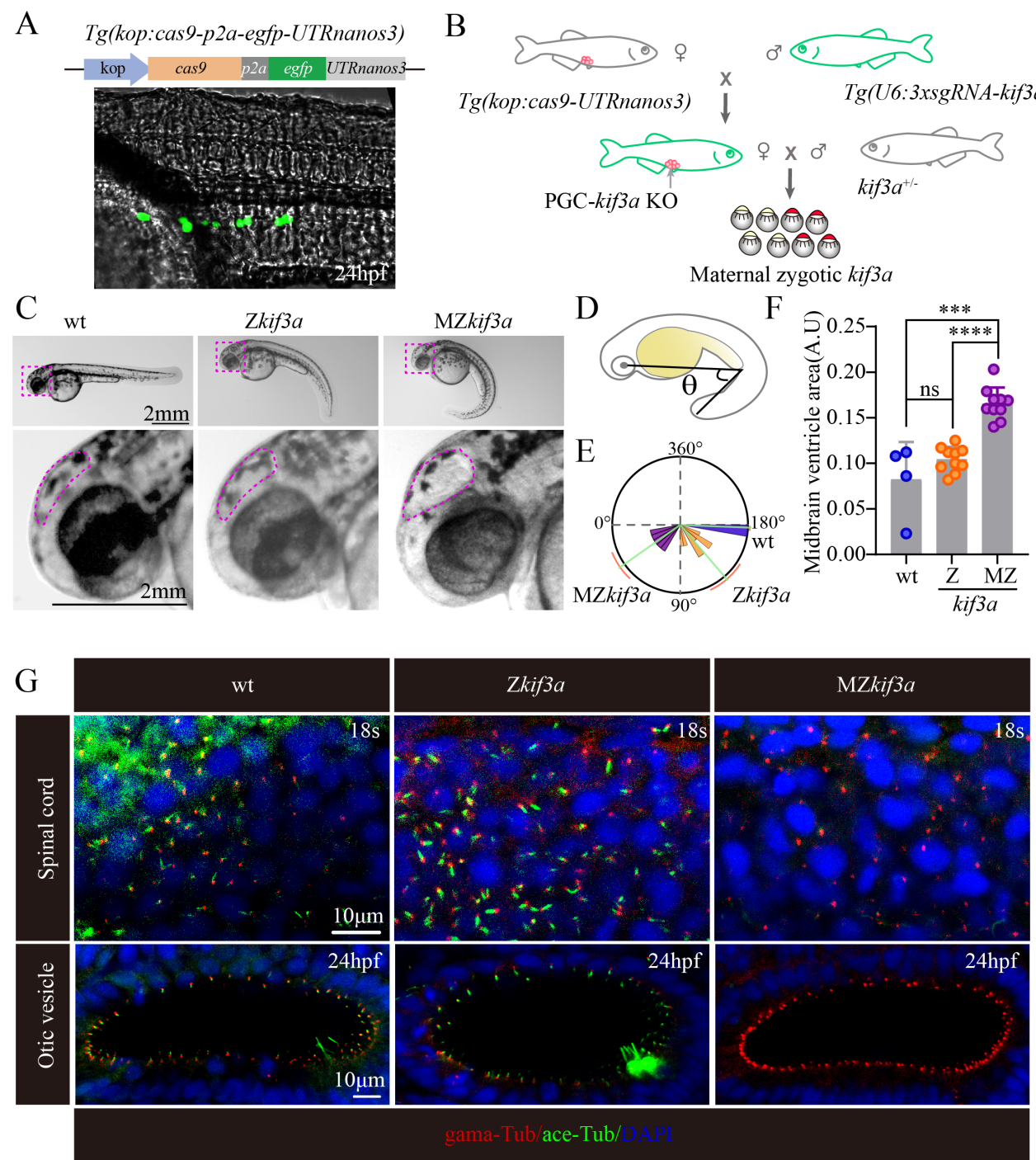

Fig 2. Generation of MZkif3a mutants via germ cell-specific expression of Cas9

(A) Bright field image showing a 24 hpf $T g(k o p$ : cas9-p2a-egfp-UTRnanos3) zebrafish embryo with EGFP fluorescence in the PGCs. Top: Diagram of the construct for making this transgene. (B) Schematic diagram showing the strategy for generating germ cell-specific knockout mutants of kif3a (gcKOkif3a). (C) External phenotypes of $24 \mathrm{hpf}$ Zkif3a and MZkif3a mutants. The enlarged boxes were shown in the bottom and dots outline the midbrain ventricles. (D-E) Statistical analysis showing the increased body curvature severity as demonstrated by the reduced angles of MZkif3a mutants. (F) Bar graph showing relative size of midbrain ventricles in different mutants as indicated. A.U. arbitrary unit. (G) Confocal images showing cilia in the spinal cord and otic vesicle of wild type, Zkif3a and MZkif3a mutants as indicated. Cilia were labeled with acetylated tubulin in green and the basal bodies were stained with $\gamma$-tubulin in red. Nuclei were counterstained with DAPI. 
A

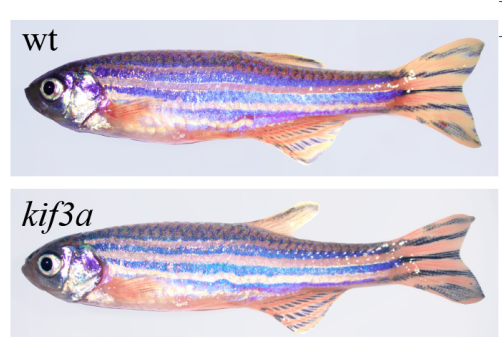

$\mathrm{C}$

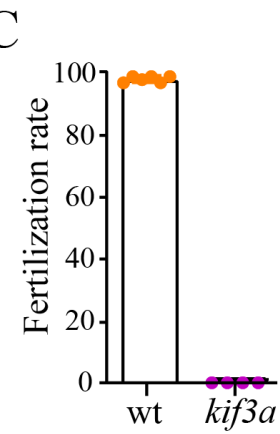

$\mathbf{F}$

$\mathrm{D}$
$\mathrm{B}$

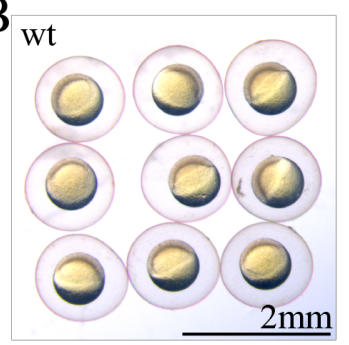

kif3a

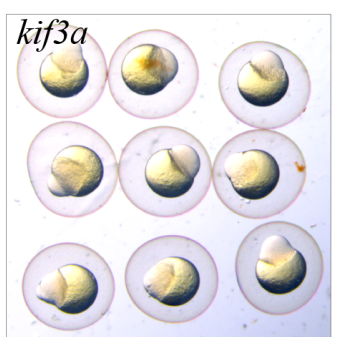

$\mathrm{E}$
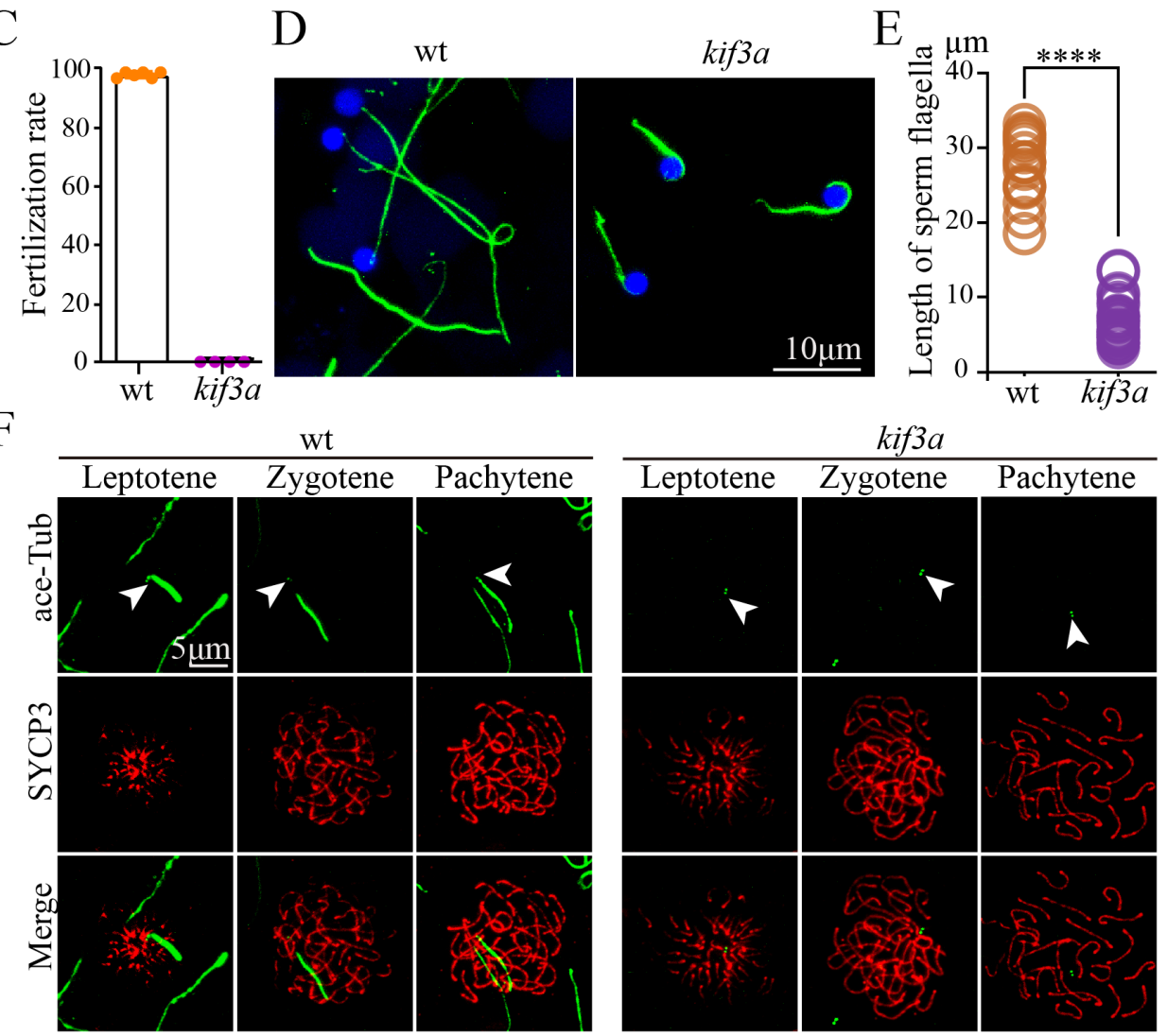

kif3a

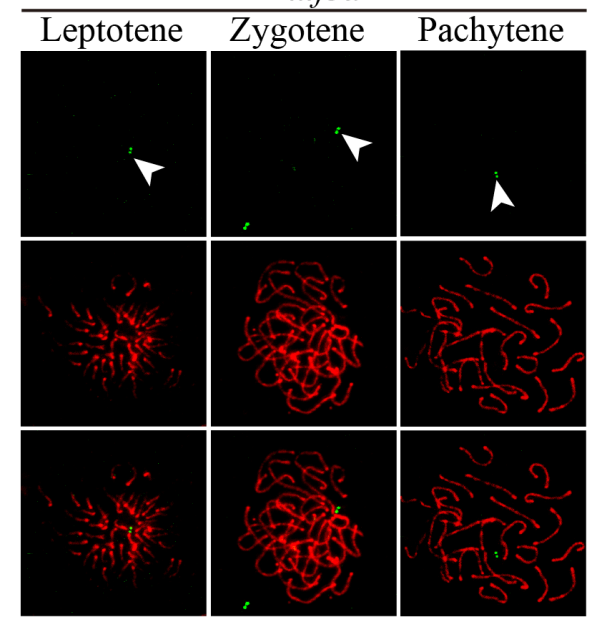

Fig 3. Phenotypes of germ cell kif3a conditional knockout mutants

(A) External phenotypes of wild type and $T g(k o p$ : cas9-UTRnanos3; U6:3xsgRNAkif3a) double transgenic male fish. (B) The embryos produced from wild type female crossed with control or double transgenic male at 6 hours after fertilization. (C) Dot plot showing the percentages of fertilization rates of wild type and double transgenic males as indicated. (D) Confocal images showing sperm flagella in wild type and double transgenic fish labeled with anti-acetylated tubulin antibody. (E) Statistical analysis of the length of sperm flagella in wild type and double transgenic fish. (F) Confocal images showing cilia in primary spermatocytes of wild type and double transgenic fish. Spermatocyte cilia (green) were absent in the double transgenic fish. The stages of spermatocytes were distinguished by the staining of SYCP3 (Red). 

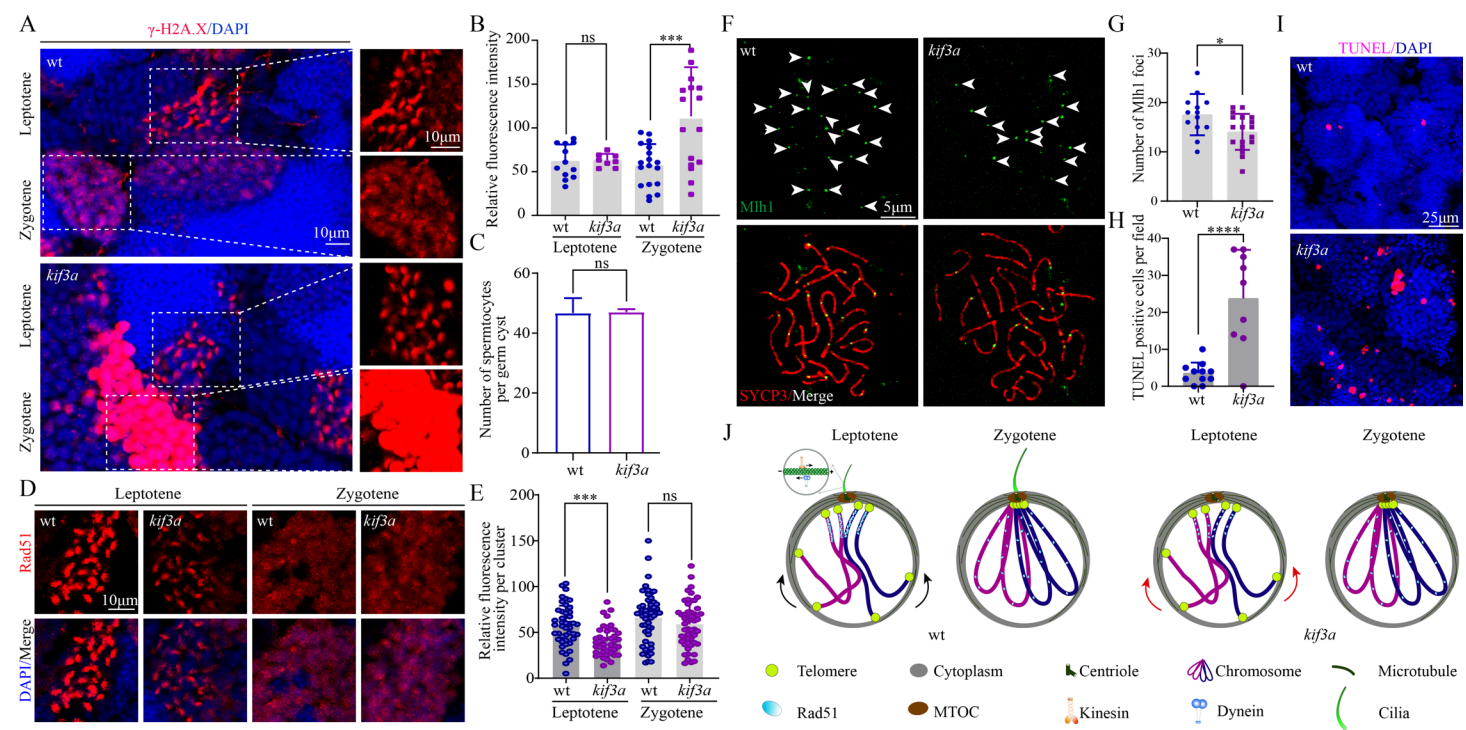

Fig 4. The repair of DSBs was compromised in the absence of spermatocyte cilia

(A) Confocal images showing the staining of $\gamma$-H2A.X (red) in the testis of wild-type and $\operatorname{Tg}$ (kop: cas9-UTRnanos3;U6:3xsgRNA-kif3a) double transgenic fish. (B) Statistical analysis showing relative fluorescence intensity of $\gamma-\mathrm{H} 2 \mathrm{~A} . \mathrm{X}$ staining on leptotene and zygotene spermatocytes of wild type and double transgenic fish. (C) Statistical analysis of the number of spermatocytes per germ cyst in wild type and double transgenic fish. (D) Staining of Rad51 in the testis of wild-type and double transgenic fish. (E) Statistical analysis of relative fluorescence intensity of Rad51staining on leptotene and zygotene spermatocytes. (F) Staining of Mlh1 in primary spermatocytes of wild-type and double transgenic fish. (G) Statistical analysis of the Mlh1 foci number per spermatocyte. (H-I) Apoptotic cells stained by TUNEL assay in wild type and double transgenic fish. (J) Model illustrating the roles of spermatocyte cilia in DSBs repair. In the absence of cilia, less Rad51 proteins are recruited to the DSBs near the MTOC, which compromises the efficiency of DSBs repair. 


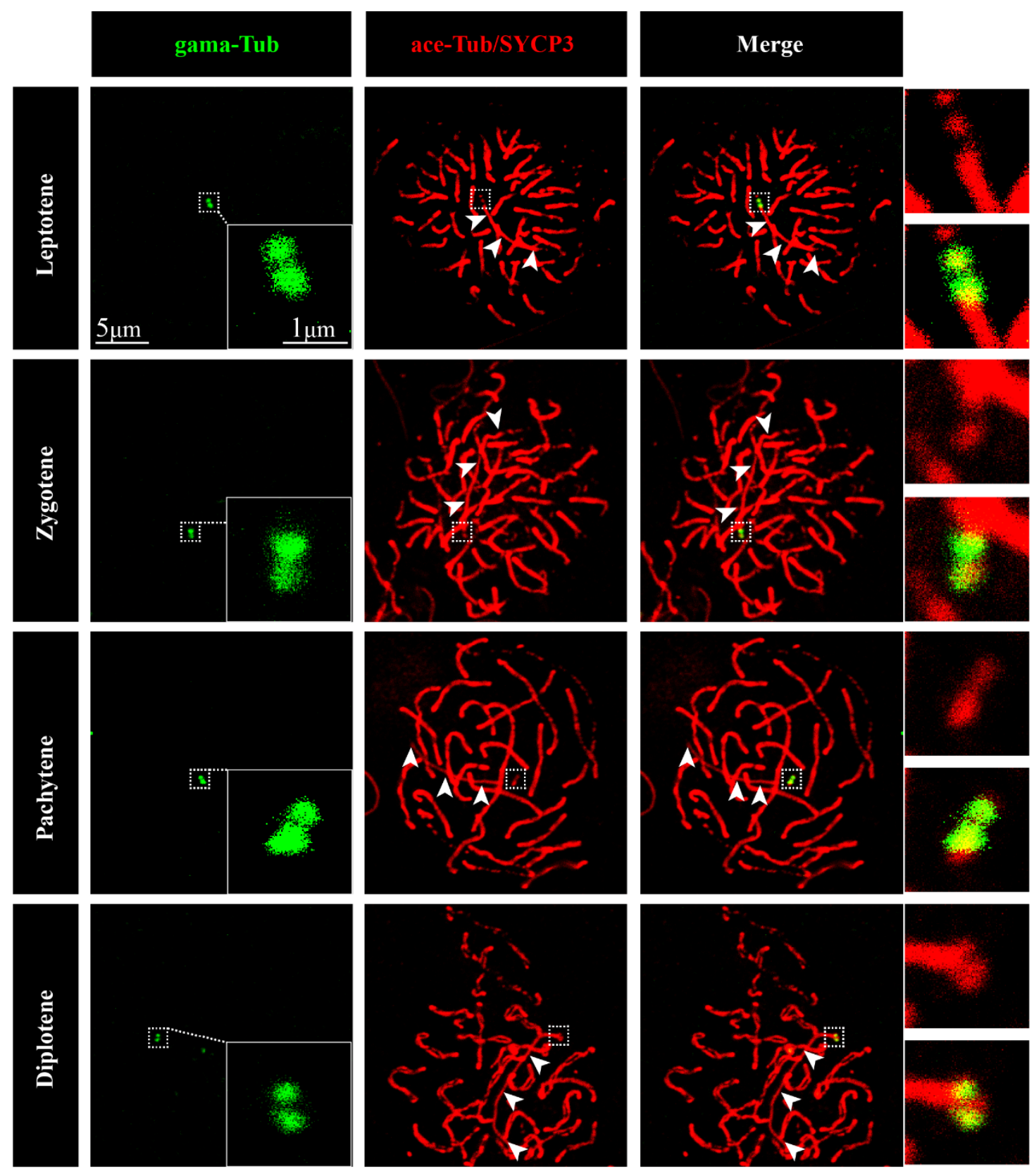

Fig S1. Cilia of primary spermatocyte at different stages

Confocal images showing basal bodies and synaptonemal complexes labeled with anti$\gamma$ tubulin (green) and anti-SYCP3 (red) antibodies at different stages of primary spermatocytes as indicated. Because both SYCP3 and acetylated-tubulin antibodies were raised in rabbit, spermatocyte cilia were also labeled in red (white arrowhead). The spermatocyte cilia can be distinguished by the basal colocalization with anti- $\gamma$ tubulin antibodies. 


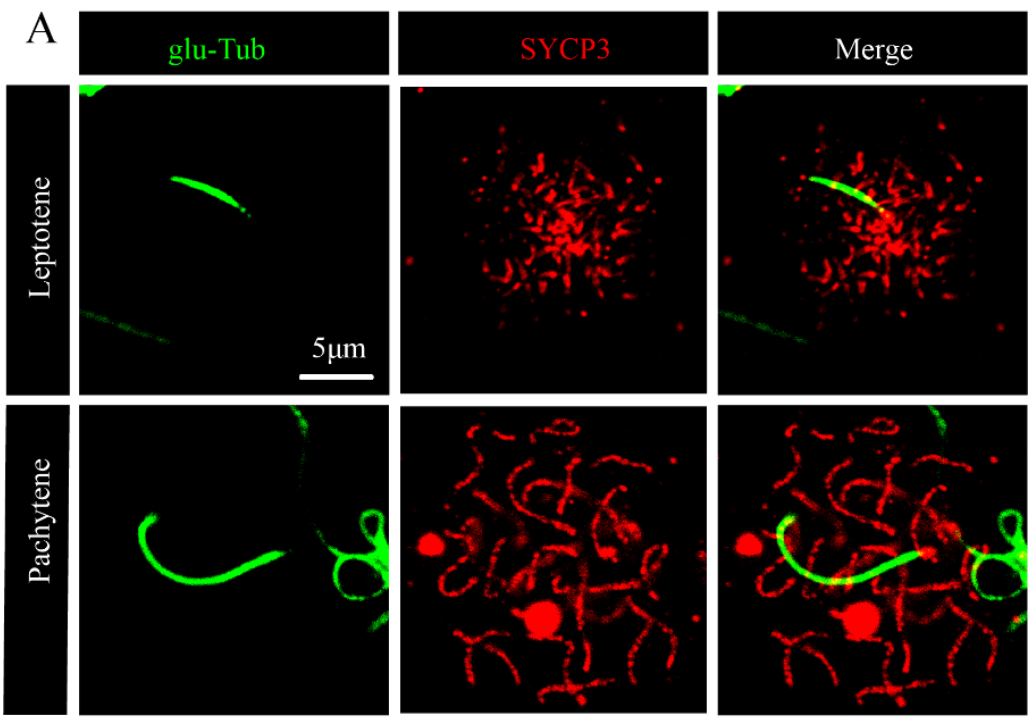

$\mathrm{B}$
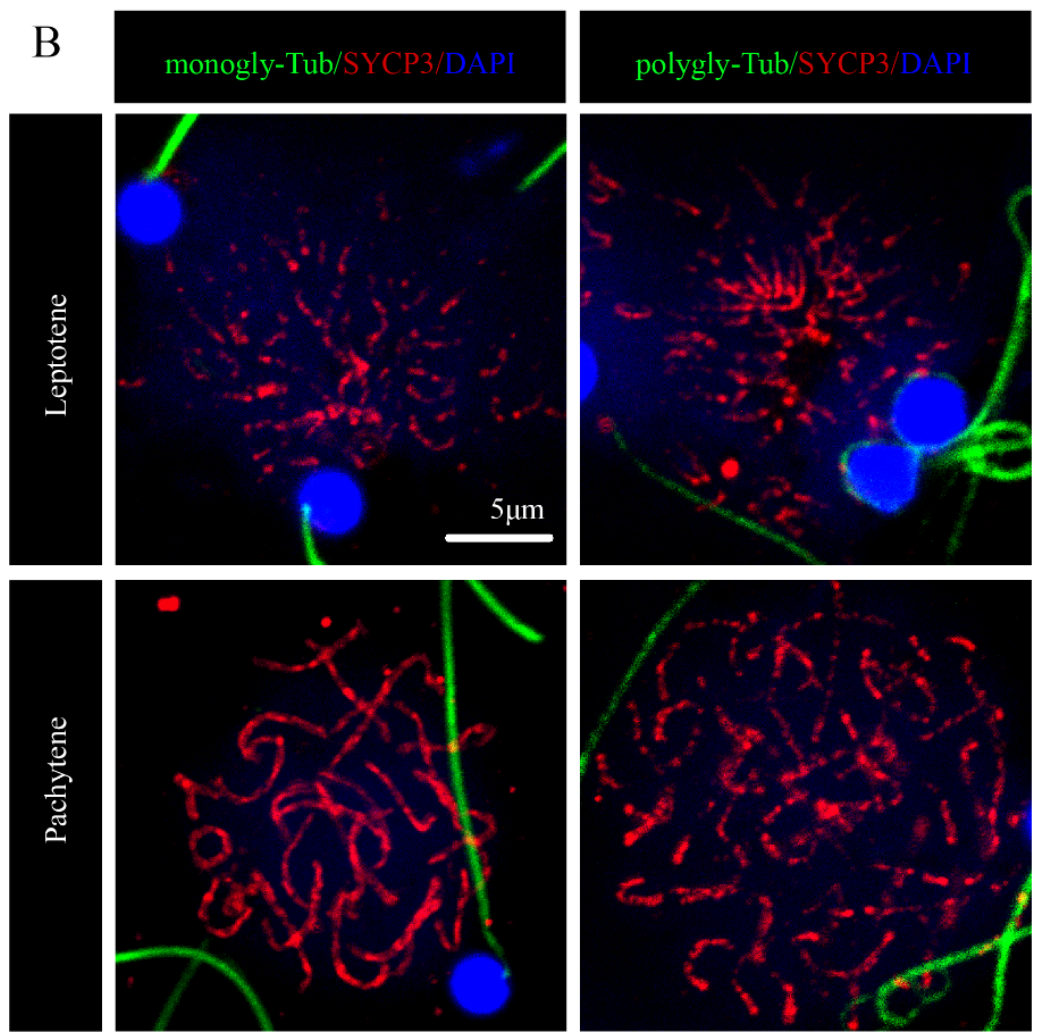

Fig S2. Tubulin modification inside spermatocyte cilia

(A) Confocal images showing cilia and synaptonemal complexes labeled with antiglutamylated-tubulin (glu-Tub, green) and anti-SYCP3 (SYCP3, red) antibodies. (B) Confocal images showing staining of mono- glycylated (monogly-Tub, green) or polyglycylated (polygly-Tub, green) Tubulin antibodies. The sperm flagella, but not spermatocyte cilia, can be labeled with these antibodies. 


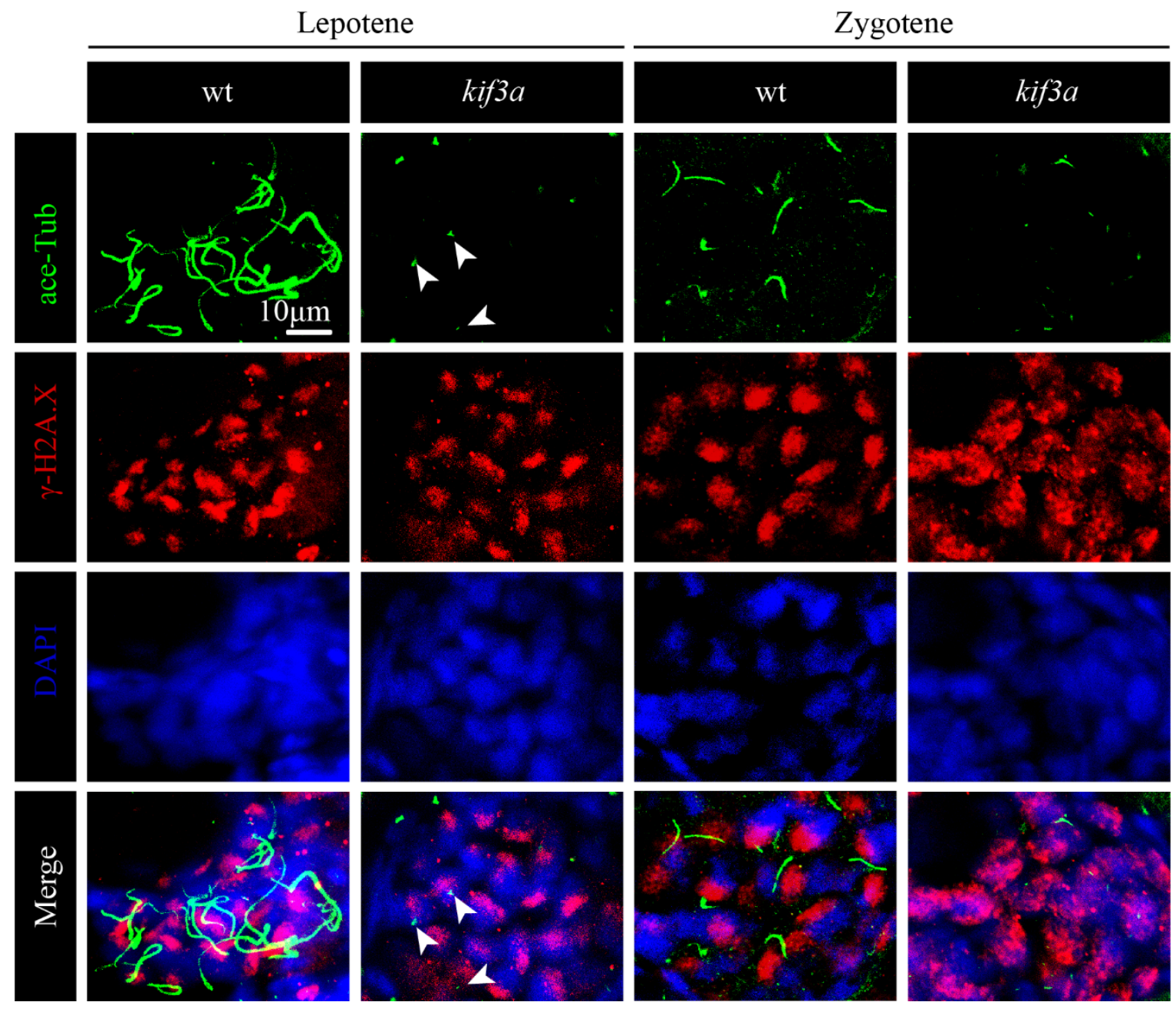

Fig S3. Cilia were present in primary oocytes

Staining of $\gamma$-H2A.X (red) and acetylated tubulin (green) in the oocytes of wild-type and $\operatorname{Tg}$ (kop:cas9-UTRnanos3; U6:3xsgRNA-kif3a) double transgenic fish. Arrowheads indicate basal bodies left in the mutant oocytes. 
A

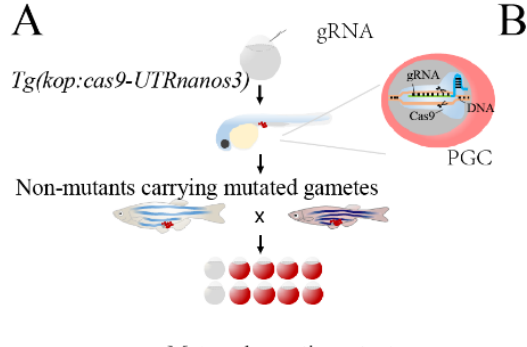

$\mathrm{D}$
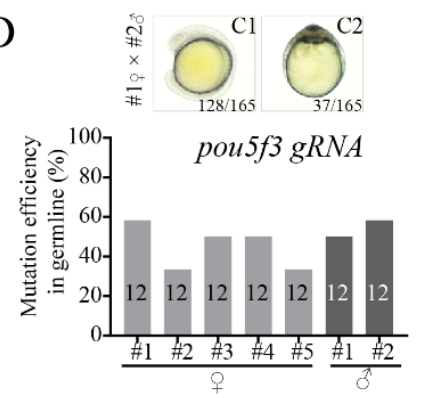

B
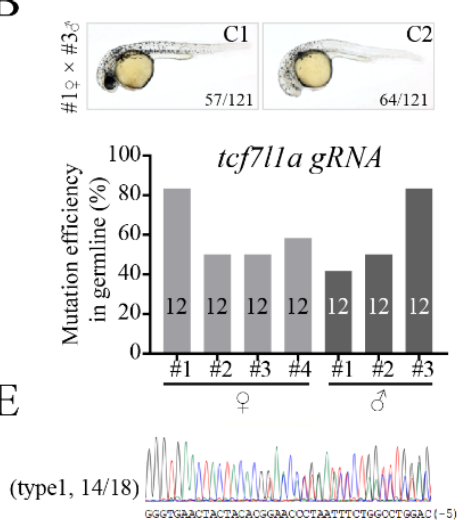

(type2, 4/18)

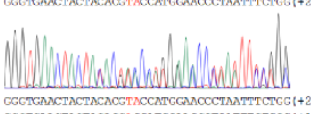

wt

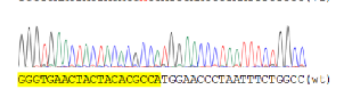

$\mathrm{C}$
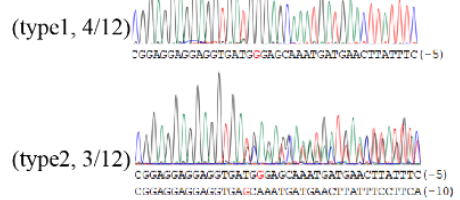

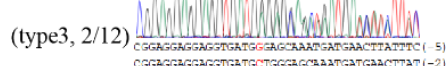

(type4, 1/12)

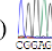

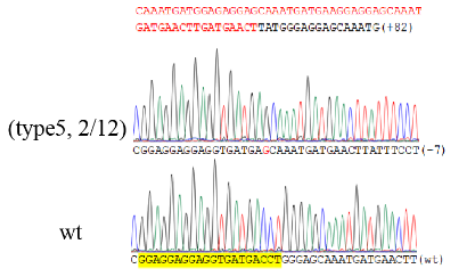

Fig S4. Generation of maternal-zygotic mutants via PGC-specific Cas9 transgenic line

(A) Schematic workflow showing process of generating MZ mutants using PGCspecific Cas9 expressing $\operatorname{Tg}$ (kop:cas9-UTRnanos3) embryos. (B) Mutation efficiencies of gametes and the phenotypes of offspring of tcf7lla sgRNA injected $\operatorname{Tg}$ (kop:cas9UTRnanos3) fish. C1 shows the WT like phenotype, C2 shows complete loss of eyes. The mutation efficiencies were calculated by the number of mutated heterozygotic embryos from crossing between F0 and wild type fish. A total of 12 embryos were genotyped from each cross. (C) The genotype of MZtcf7lla with C2 phenotype in panel B. (D) Mutation efficiencies of gametes and the phenotypes of offspring of pou $5 f 3$ sgRNA injected Tg(kop:cas9-UTRnanos3) fish. C1 shows the WT like phenotype, C2 shows the phenotype of MZpou5f3. (E)The genotype of MZpou5f3 with C2 phenotype in panel D. 

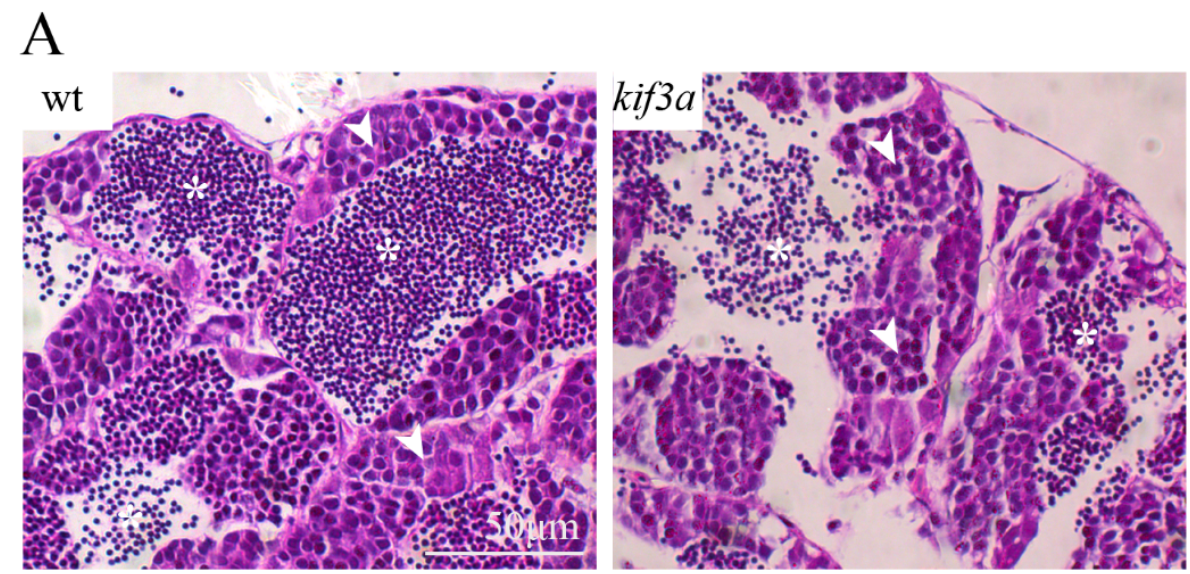

$\mathrm{B}$

wt
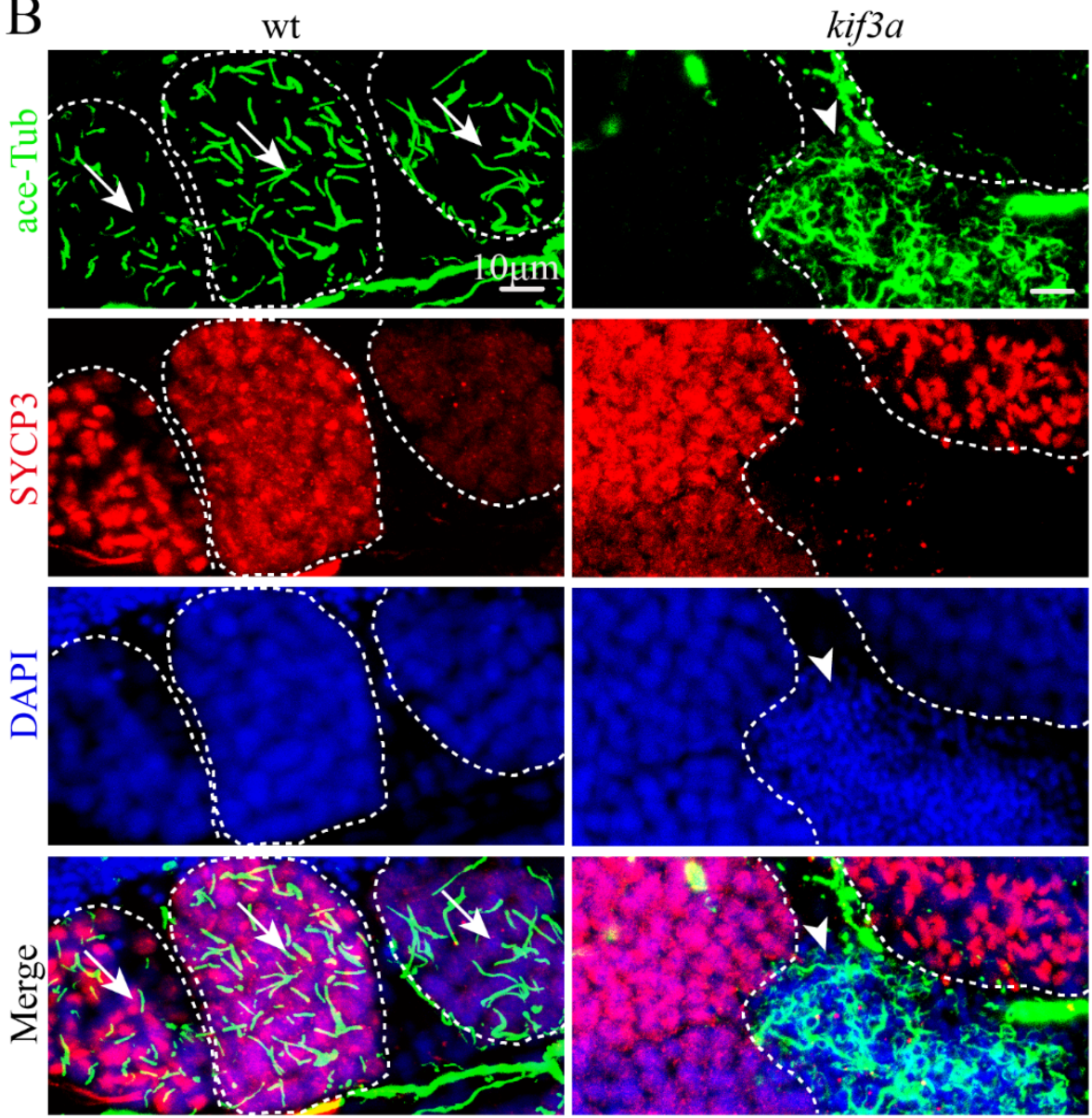

Fig S5. Germ cell-specific Kif3a conditional knockout inhibited the formation of spermatocyte cilia

(A) Histological analysis of testis of wild type and $\operatorname{Tg}$ (kop:cas9-UTRnanos; U6:3xsgRNA-kif3a) double transgenic fish. Arrowheads point to spermatocyte and asterisks indicate spermatozoa. (B) Staining of SYCP3 (red) and acetylated-tubulin (green) in the testis of wild-type and double transgenic fish. Arrows indicate spermatocyte cilia, while arrowheads indicate the staining of abnormal sperm flagella in the mutant testis. 

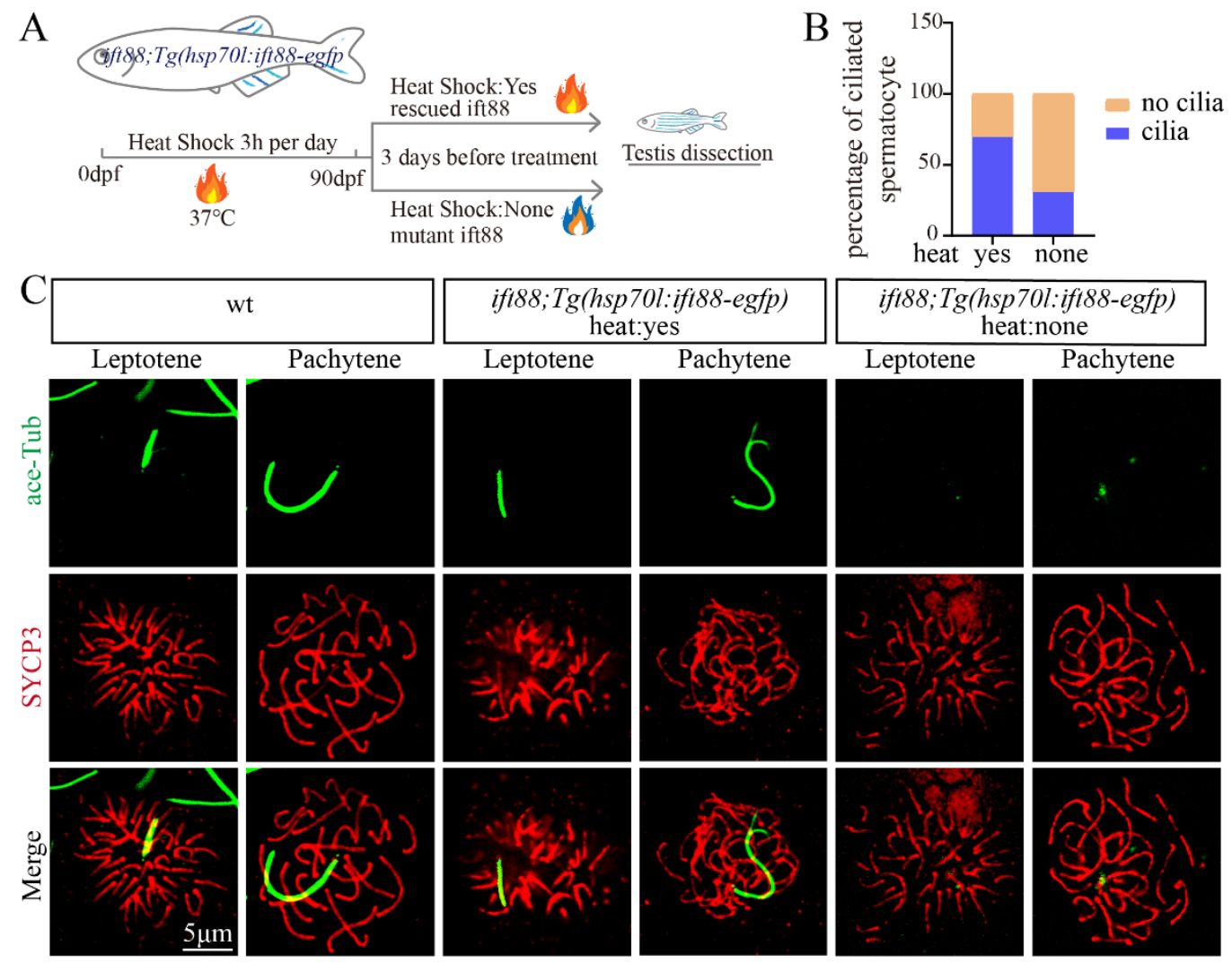

D
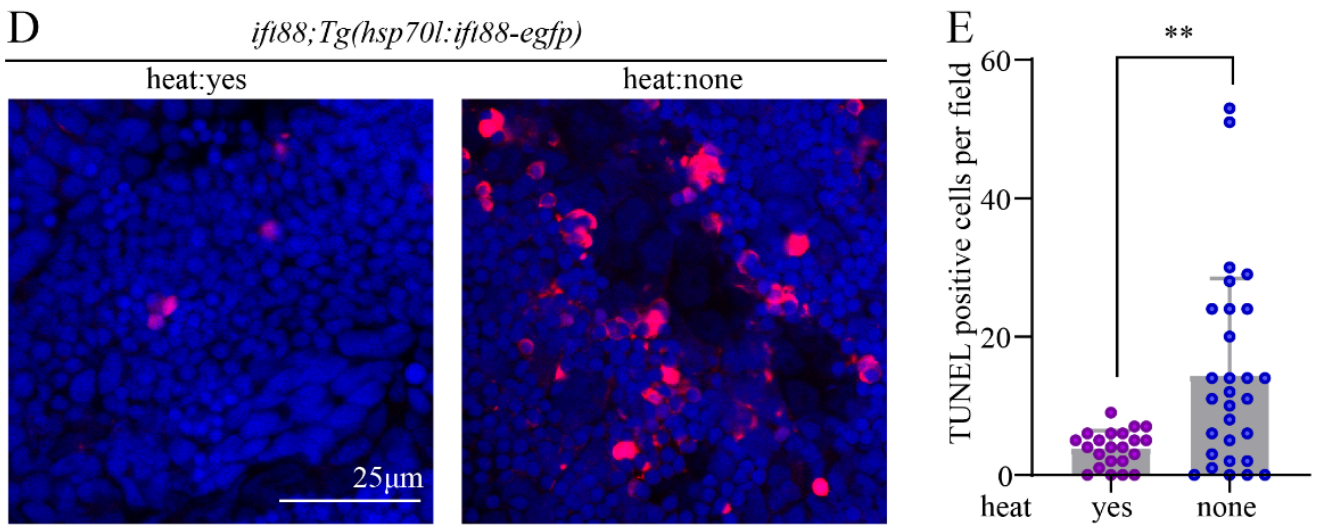

Fig S6. Ift88 deficiency resulted in ciliogenesis defects and apoptosis of spermatocytes

(A) Schematic workflow showing the strategy of generating $\operatorname{Tg}$ (hsp70l:ift88-egfp) transgene to rescue ovl(ift88) mutants. (B) Statistical analysis of the percentage of ciliated spermatocytes in heat-shocked and non-heat-shocked ift 88 ; $\operatorname{Tg}$ (hsp70l:ift88egfp) transgenic fish. (C) Confocal images showing cilia and synaptonemal complexes labeled with anti-acetylated tubulin (green) and anti-SYCP3 (red) antibodies on primary spermatocytes from different fish as indicated. (D-E) Confocal images showing apoptotic cells stained by TUNEL assay (D) and the statistical results (E) of TUNEL positive cells in heat-shocked and non-heat-shocked fish as indicated. 
A
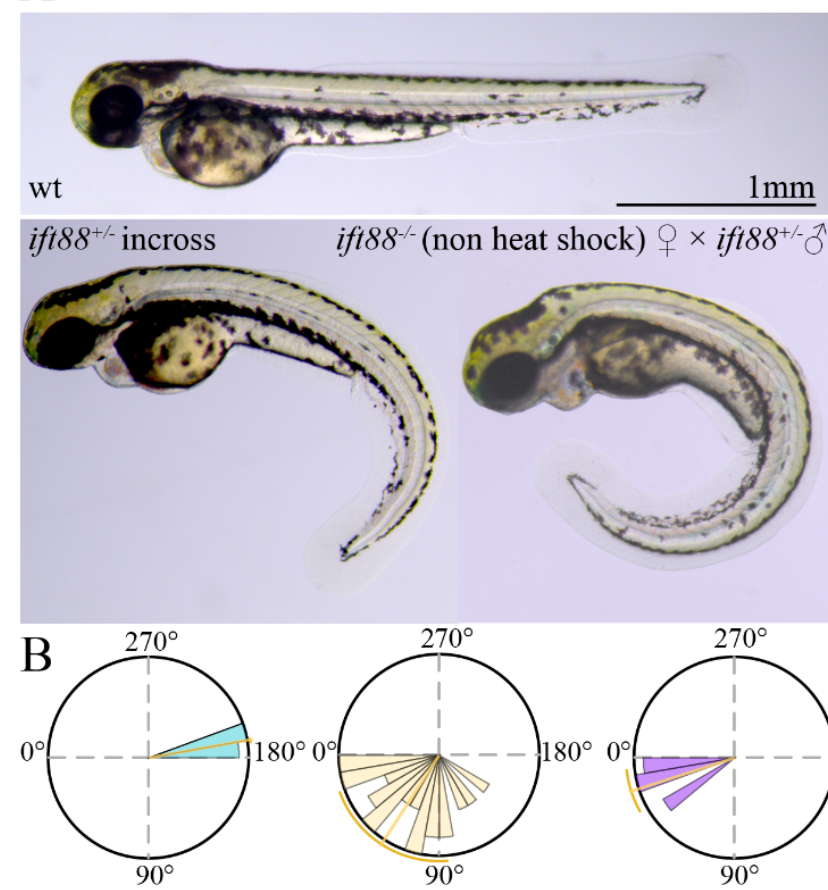

wt

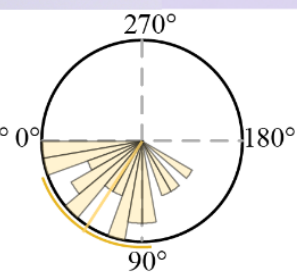

ift $88^{+/}$incross

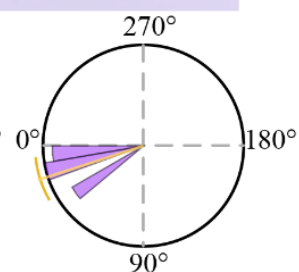

ift $88^{-/}$(non heat shock) ?
$\mathrm{C}$

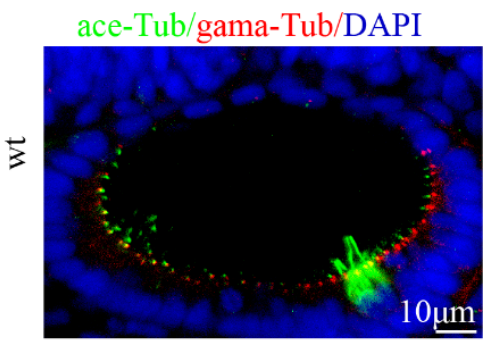

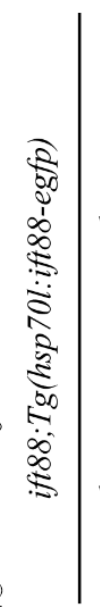

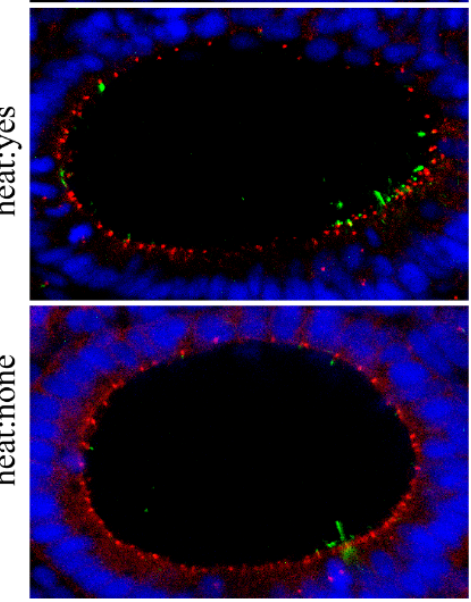

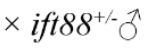

\section{Fig S7. Phenotypes of ift88 mutants}

(A) External phenotypes of wild type and ift 88 mutants generated from different crosses as indicated. (B) Statistical analysis of the angles of body curvature in wild type and ift 88 mutants as indicated. (C) Confocal images showing cilia in otic vesicle of 18somite stage wild type and ift 88 mutants as indicated. 